\title{
Necessity of the integration Internet of Things and cloud services with quality of service assurance approach
}

\author{
Hourieh KHODKARI $^{1}$, Saied Ghazi MAGHREBI ${ }^{2}$ \\ ${ }^{1} \mathrm{PhD}$ student in Computer Engineering from the Islamic Azad University, Science and \\ Research Branch, Tehran, Iran- Khodkari@gmail.com \\ ${ }^{2}$ Assistant of Professor in Islamic Azad University, Yadegar Imam Khomeini (RAH)Shahr-e- \\ Rey Branch, Tehran, Iran - GhaziMaghrebi@jdnasir.ac.ir
}

\begin{abstract}
IoT is generally characterized by real world small things, widely distributed, with limited storage and processing capacity, which involve concerns regarding reliability, performance, security, and privacy. On the other hand, Cloud computing has virtually unlimited capabilities in terms of storage and processing power, is a much more mature technology, and has most of the IoT issues at least partially solved. Thus, a novel IT paradigm in which Cloud and IoT are two complementary technologies merged together is expected to disrupt both current and Future Internet. We call this new paradigm CloudIoT. Therefore, the integration of cloud and Internet of Things to providing the best service to users and have the highest level of customer satisfaction should always provide quality of service can be guaranteed. The purpose of this paper is to evaluate the integrity requirements of cloud services and Internet of Things so that quality of service in these services must be guaranteed.
\end{abstract}

Keywords: Cloud Services, Internet of Things, Quality of Service, Service Level Agreement, Cloud of Things, Integration.

\section{Introduction}

The Internet of Things (IoT), also called the Internet of Everything or the Industrial Internet, is a new technology paradigm envisioned as a global network of machines and devices capable of interacting with each other. The IoT is recognized as one of the most important areas of future technology and is gaining vast attention from a wide range of industries. The true value of the IoT for enterprises can be fully realized when connected devices are able to communicate with each other and integrate with vendor-managed inventory systems, customer support systems, business intelligence applications, and business analytics [1].

In this paper, we focus our attention on the integration of Cloud and IoT, which is what we call the CloudIoT paradigm. The Internet of Things (IoT) paradigm is based on intelligent and 
self-configuring nodes (things) interconnected in a dynamic and global network infrastructure [2]. Introduction of the new technologies cloud computing and Internet of Things discuss in section 1. In Section 2 we describe about the Cloud computing and. In Section 3 we discuss about Internet of Things (IoT). The integration of Cloud computing and Internet of Things (IoT) that called the ClouldIoT, discuss in section 4. Quality of service in the ClouldIoT describe in section 5, and conclusions in section 6 represent.

\section{Cloud Computing}

Cloud Computing enables a convenient, on demand and scalable networks access to a pool of configurable computing resources. Cloud Computing has virtually unlimited capabilities in terms of storage and processing power.

Here are some definitions of cloud computing:

- Cloud computing is a model for enabling convenient, on-demand network access to a shared pool of configurable computing resources (e.g. networks, servers, storage, applications and services) that can be rapidly provisioned and released with minimal management effort or service provider interaction. (National Institute of Standards and Technology (NIST)) [3,4].

- A style of computing where massively scalable IT-enabled capabilities are delivered 'as a service' to external customers using Internet technologies. (Gartner) [5]

- An emerging IT development, deployment and delivery model, enabling real-time delivery of products, services and solutions over the Internet. (IDC) [6]

- 'Cloud computing' describes a service model that combines a general organizing principle for IT delivery, infrastructure components, an architectural approach and an economic model - basically, a confluence of grid computing, virtualization, utility computing, hosting and software as a service (SaaS). (The Group 451)

However, each research group defines cloud computing by its own understanding and approaches so that it is very difficult to give the most general definition. Cloud computing is typically classified in the following four ways:

- Public cloud: In Public cloud the computing infrastructure is hosted by the cloud vendor. The customer has no visibility and control over where the computing infrastructure is hosted. The computing infrastructure is shared between any organizations. 
Bulletin de la Société Royale des Sciences de Liège, Vol. 85, 2016, p. 434 - 445

- Private cloud: The computing infrastructure is dedicated to a particular organisation and not shared with other organisations. Some experts consider that private clouds are not real examples of cloud computing. Private clouds are more expensive and more secure when compared to public clouds.

- Hybrid cloud Organisations may host critical applications on private clouds and applications with relatively less security concerns on the public cloud. The usage of both private and public clouds together is called hybrid cloud.

- Community Cloud: is type of cloud hosting in which the setup is mutually shared between many organisations that belong to particular community, i.e. banks and trading firms. It is multi-tenant setup that is shared among several organisations that belong to a specific group which has similar computing apprehensions.

Cloud Computing is a disruptive technology with profound implications for the delivery of Internet services as well as for the IT sector as a whole. However, several technical and business-related issues are still unsolved. Specific issues have been identified for each service models, which are mainly related to security (e.g., data security and integrity, network security), privacy (e.g., data confidentiality), and service-level agreements, which could scare away part of potential users [2].

Three types of cloud computing as shown in Figure 1 are summarized as follows:

- IaaS (Infrastructure-as-a-Service)

- PaaS (Platform-as-a-Service)

- SaaS (Software-as-a-Service)

Cloud computing applies a utility model to produce and consume computing resources, in which the Cloud abstracts all types of computing resources, including storage, as services (i.e. Cloud services). The Cloud user (either application developer or application consumer) can access the Cloud services over the Internet, and the Cloud users pay only for time and services they need. The Cloud can also scale to support large numbers of service requests. Ultimately, Cloud computing takes care of the micro-lifecycle management of applications, and allows application managers to focus on application development and monitoring. The Cloud computing platform is designed to consist of a variety of services for developing, testing, running, deploying, and maintaining applications on the Cloud. Examples of Cloud computing platforms are The Amazon Web Services, Google App Engine, and Microsoft's Windows Azure platform [8]. 


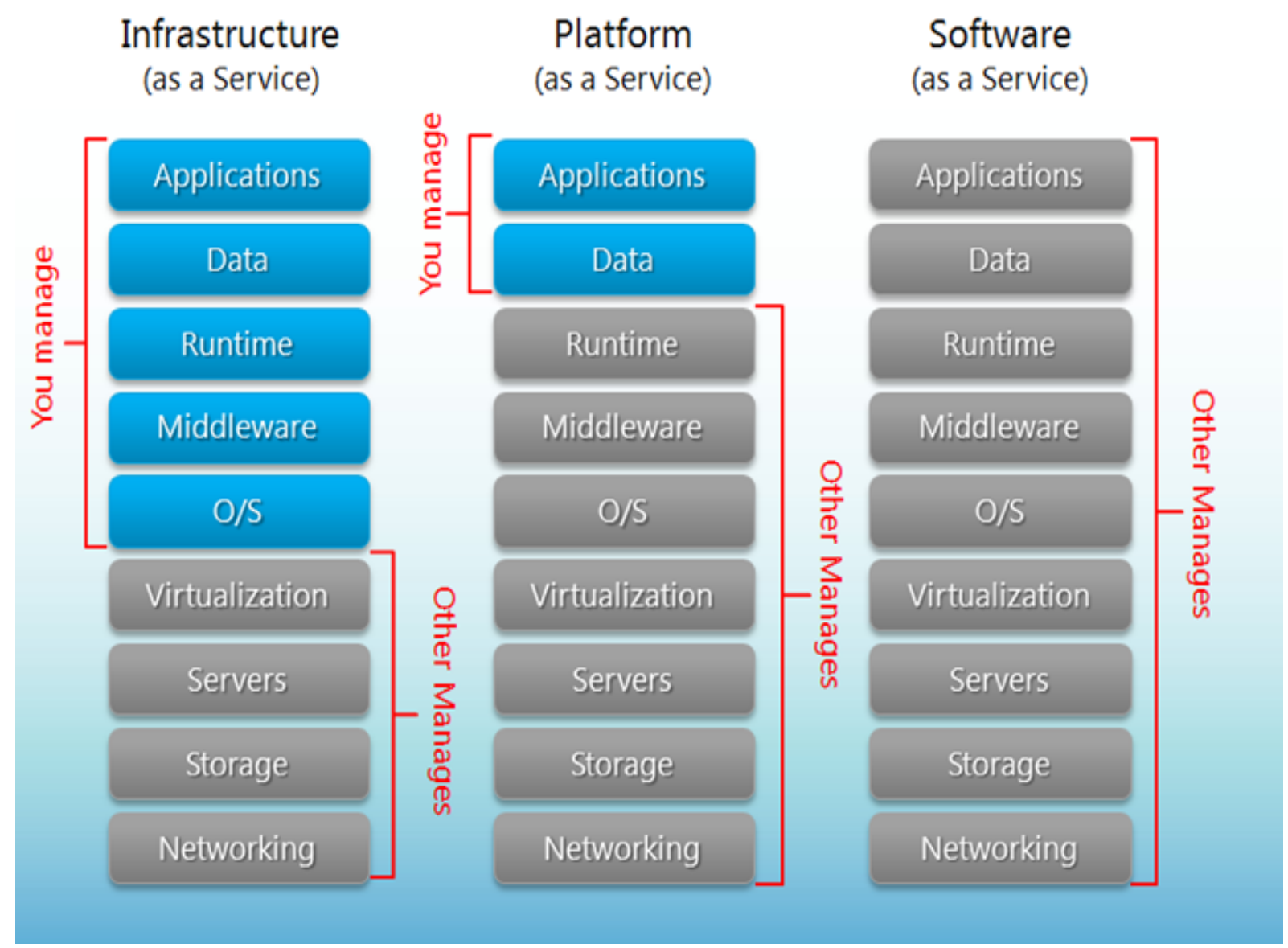

Fig. 1: Types of cloud computing

\section{Internet of Things}

IoT, the term first introduced by Kevin Ashton in 1999, is a future of Internet and ubiquitous computing. This technological revolution represents the future of connectivity and reachability. In IoT, 'things' refer to any object on face of the Earth, whether it is a communicating device or a non-communicating dumb object. Although the definition of 'Things' has changed as technology evolved, the main goal of making a computer sense information without the aid of human intervention remains the same. A radical evolution of the current Internet into a Network of interconnected objects that not only harvests information from the environment (sensing) and interacts with the physical world (actuation/command/control), but also uses existing Internet standards to provide services for information transfer, analytics, applications, and communications.

IoT refers to "a world-wide network of interconnected objects uniquely addressable, based on standard communication protocols"' whose point of convergence is the Internet. The basic idea behind it is the pervasive presence around people of things, able to measure, infer, 
understand, and even modify the environment. IoT is fueled by the recent advances of a variety of devices and communication technologies, but things included in IoT are not only complex devices such as mobile phones, but they also comprise everyday objects such as food, clothing, furniture, paper, landmarks, monuments, works of art, etc. [9]. These objects, acting as sensors or actuators, are able to interact with each other in order to reach a common goal. The key feature in IoT is, without doubt, its impact on everyday life of potential users [10]. IoT has remarkable effects both in work and home scenarios, where it can play a leading role in the next future (assisted living, e-health, smart transportation, etc.). Important consequences are also expected for business (e.g. logistic, industrial automation, transportation of goods, security, etc.). According to these considerations, in 2008 IoT has been reported by US National Intelligence Council as one of the six technologies with potential impact on US interests towards 2025 [11]. Indeed, in 2011 the number of interconnected devices overtook the number of people. In 2012, the number of interconnected devices was estimated to be 9 billion, and it was expected to reach the value of 24 billion by 2020. Such numbers suggest that IoT will be one of the main sources of big data [12]. In the following we describe a few important aspects related to IoT [2]:

- $\quad$ RFID. In IoT scenario, a key role is played by Radio-Frequency Identification (RFID) systems, composed of one or more readers and several tags. These technologies help in automatic identification of anything they are attached to, and allow objects to be assigned unique digital identities, to be integrated into a network, and to be associated with digital information and services [9]. In a typical usage scenario, readers trigger the tag transmission by generating an appropriate signal, querying for possible presence of objects uniquely identified by tags. RFID tags are usually passive (they do not need on-board power supply), but there are also tags powered from batteries [10, 13].

- (Wireless) sensor networks. Another key component in IoT environments is represented by sensor networks. For example, they can cooperate with RFID systems to better track the status of things, getting information about position, movement, temperature, etc. Sensor networks are typically composed of a potentially high number of sensing nodes, communicating in a wireless multi-hop fashion. Special nodes (sinks) are usually employed to gather results. Wireless sensor networks (WSNs) may provide various useful data and are being utilized in several areas like healthcare, government and environmental services (natural disaster relief), defense (military 
target tracking and surveillance), hazardous environment exploration, seismic sensing, etc. [14]. However, sensor networks have to face many issues regarding their communications (short communication range, security and privacy, reliability, mobility, etc.) and resources (power considerations, storage capacity, processing capabilities, bandwidth availability, etc.). Besides, WSN has its own resource and design constraints (that are application- and environment- specific) and that heavily depend on the size of the monitoring environment [14].

- Addressing. Thanks to wireless technologies such as RFID and Wi-Fi, IoT paradigm is transforming the Internet into a fully integrated Future Internet [13]. While Internet evolution led to an unprecedented interconnection of people, current trend is leading to the interconnection of objects, to create a smart environment [15]. In this context, the ability to uniquely identify things is critical for the success of IoT since this allows to uniquely address a huge number of devices and control them through the Internet. Uniqueness, reliability, persistence, and scalability represent critical features related to the creation of a unique addressing schema [15]. Unique identification issues may be addressed by IPv4 to an extent (usually a group of cohabiting sensor devices can be identified geographically, but not individually). IPv6, with its Internet Mobility attributes, can mitigate some of the device identification problems and is expected to play an important role in this field.

- Middleware. Due to the heterogeneity of the participating objects, to their limited storage and processing capabilities and to the huge variety of applications involved, a key role is played by the middleware between the things and the application layer, whose main goal is the abstraction of the functionalities and communication capabilities of the devices. The middleware can be divided in a set of layers (see Fig. 2): Object Abstraction, Service Management, Service Composition, and Application [9]. 


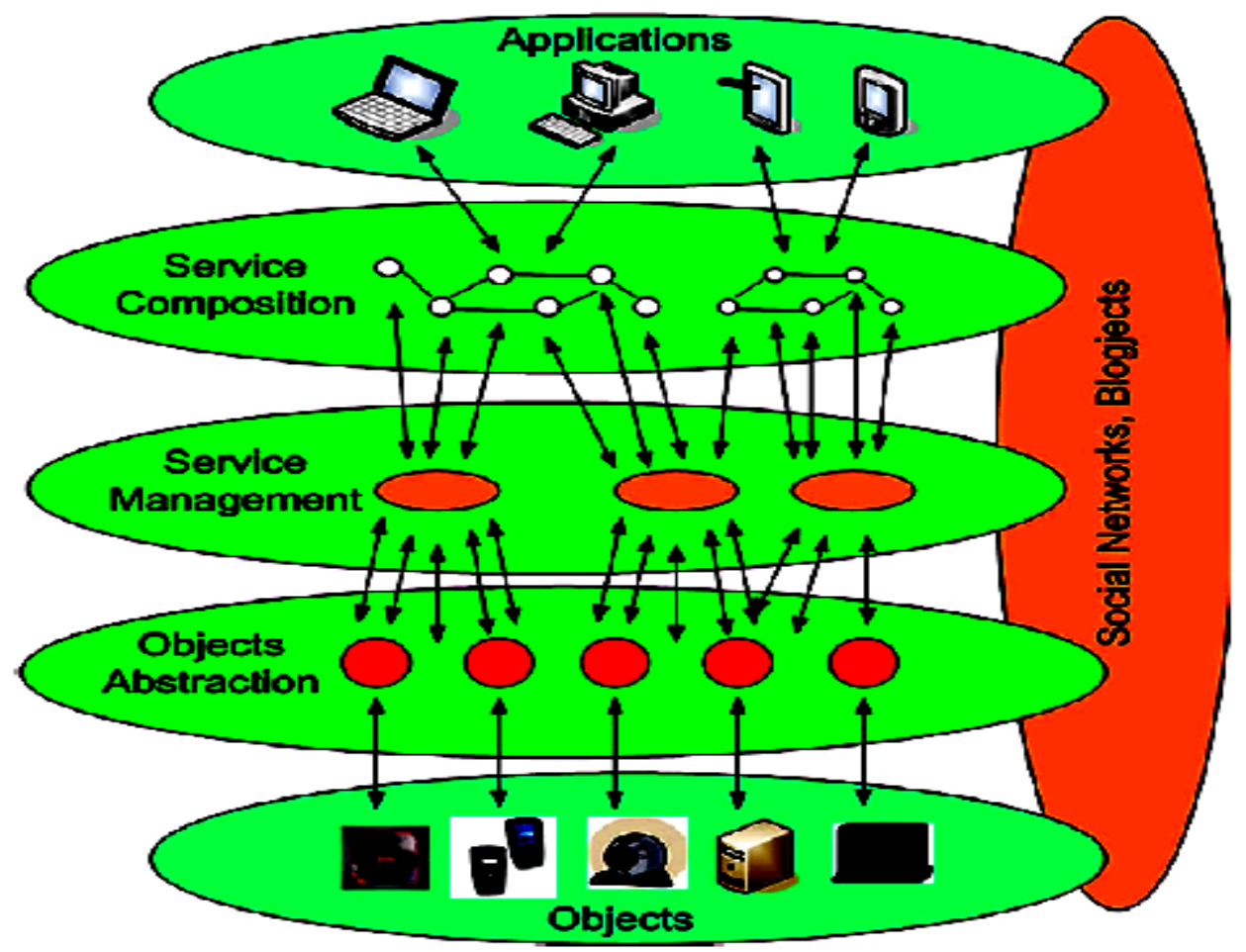

Fig. 2: IoT paradigm: an overall view [2].

\section{Integration of cloud and Internet of Things}

IoT and cloud computing working in integration makes a new paradigm, which we have termed here as CloudIoT. The two worlds of Cloud and IoT have seen an independent evolution. However, several mutual advantages deriving from their integration have been identified in literature and are foreseen in the future. On the one hand, IoT can benefit from the virtually unlimited capabilities and resources of Cloud to compensate its technological constraints (e.g., storage, processing, and energy). Specifically, the Cloud can offer an effective solution to implement IoT service management and composition as well as applications that exploit the things or the data produced by them. On the other hand, the Cloud can benefit from IoT by extending its scope to deal with real world things in a more distributed and dynamic manner, and for delivering new services in a large number of real life scenarios. These worlds are very different from each other and, even better, their characteristics are often complementary, as Table 1 shows. Such complementarity is the main reason why many researchers have proposed and are proposing their integration, generally to obtain benefits in specific application scenarios [16]. 
Table 1: Complementary aspects of Cloud and IoT [2].

\begin{tabular}{|c|c|c|}
\hline & \multicolumn{2}{|c|}{ IoT } \\
\hline Displacement & Pervasive & Cloud \\
\hline Reachability & Limited & Ubiquitous \\
\hline Components & Real world things & Virtual resources \\
\hline Computational capabilities & Limited & Virtually unlimited \\
\hline Storage & Limited or none & Virtually unlimited \\
\hline Role of the Internet & Point of convergence & $\begin{array}{c}\text { Means for delivering } \\
\text { services }\end{array}$ \\
\hline Big data & & Means to manage \\
\hline
\end{tabular}

Most of the papers in literature are actually seeing Cloud as the missing piece in the integrated scenario, i.e. they believe that Cloud fills some gaps of IoT (e.g. the limited storage). A few others, instead, see IoT filling gaps of Cloud (mainly the limited scope) [2].

Being IoT characterized by a very high heterogeneity of devices, technologies, and protocols, it lacks different important properties such as scalability, interoperability, flexibility, reliability, efficiency, availability, and security. Indeed, Cloud facilitates the flow between IoT data collection and data processing, and enables rapid setup and integration of new things, while maintaining low costs for deployment and for complex data processing [18]. As a consequence, analyses of unprecedented complexity [17] are possible, and datadriven decision making and prediction algorithms can be employed at low cost, providing means for increasing revenues and reduced risks [19].

CloudIoT gave birth to a new set of smart services and applications that can strongly impact everyday life (Fig. 3). Many of the applications described in the following (may) benefit from Machine-to-Machine communications (M2M) when the things need to exchange information among themselves and not only send them towards the cloud. These applications are the following [2]:

- Healthcare

- Smart cities and communities

- Smart home and smart metering

- Video surveillance

- Automotive and smart mobility

- Smart energy and smart grid

- Smart logistics

- Environmental monitoring 


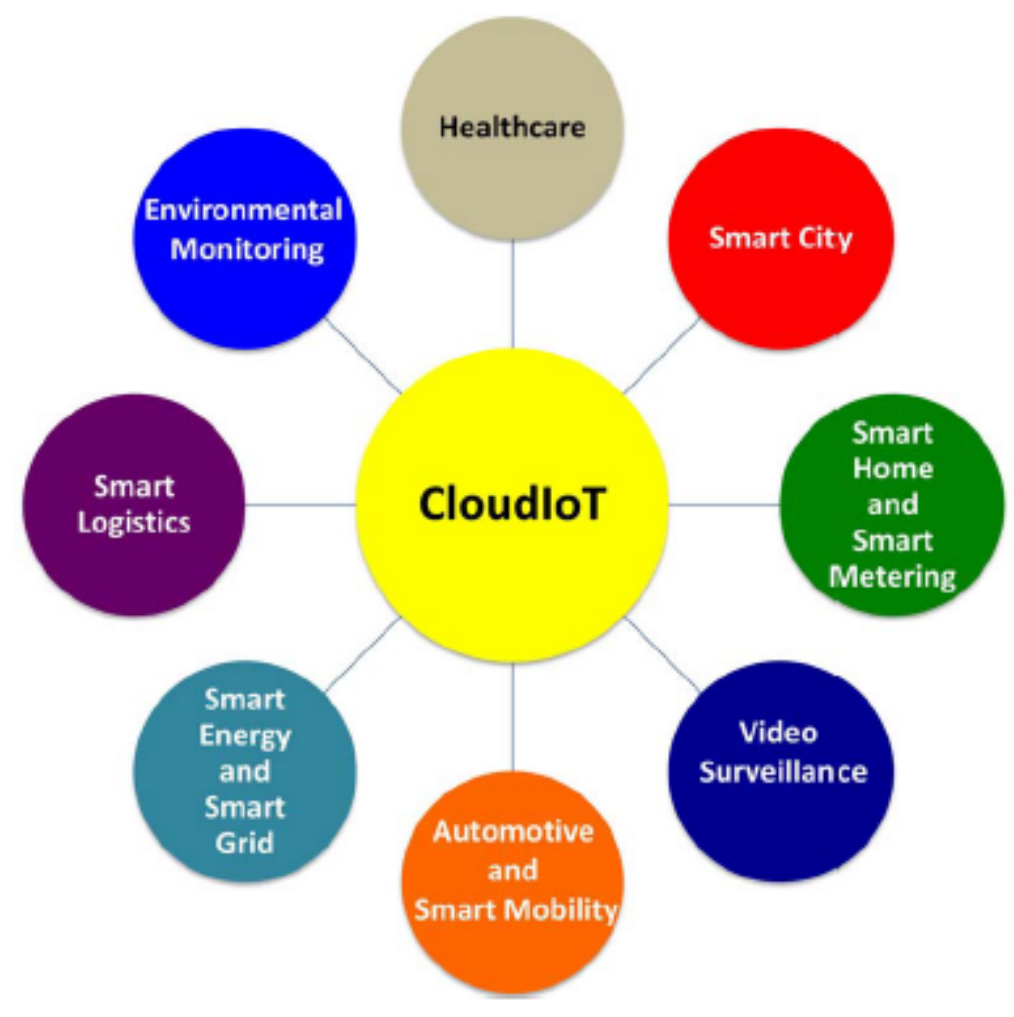

Fig. 3: Application scenarios driven by the CloudIoT paradigm [2].

\section{Quality of service In Integration of cloud and Internet of Things}

Heterogeneous networks are (by default) multi-service; providing more than one distinct application or service. This implies not only multiple traffic types within the network, but also the ability of a single network to support all applications without Quality of Service (QoS) compromise. There are two application classes: throughput and delay tolerant elastic traffic of (e.g. monitoring weather parameters at low sampling rates), and the bandwidth and delay sensitive inelastic (real-time) traffic (e.g. noise or traffic monitoring), which can be further discriminated by data-related applications (e.g. high-vs.-low resolution videos) with different QoS requirements. Therefore, a controlled, optimal approach to serve different network traffics, each with its own application QoS needs is required. It is not easy to provide QoS guarantees in wireless networks, as segments often constitute 'gaps' in resource guarantee due to resource allocation and management ability constraints in shared wireless media. Quality of Service in Cloud computing is another major research area which will require more and more attention as the data and tools become available on clouds. Dynamic scheduling and resource allocation algorithms based on particle swarm optimization are being 
developed. For high capacity applications and as IoT grows, this could become a bottleneck [15].

However, though cloud computing has tremendous advantages; there are challenges in the area of Quality of Service (QoS). QoS denotes the levels of performance, reliability and availability offered by an application and by the platform or infrastructure that hosts it. QoS is fundamental for cloud users, who expect providers to deliver the advertised quality characteristics, and for cloud providers, who need to find the right tradeoffs between QoS levels and operational costs. Any violation of service level agreement (SLA) entails a loss for both cloud providers and cloud users. Over provision is often adopted by providers as an approach to satisfy the SLA, but it fails to optimize the resource utilization, especially for private clouds.

The current mechanisms for QoS provisioning and supporting service level agreements in IoT and clouds have major limitations. In the light of CloudIoT, these mechanisms will have to be radically reconsidered or even reinvented to meet the challenges posed by upcoming CloudIoT applications. However, QoS guarantee for the CloudIoT is expectedly challenging, and an emerging discipline. This is due to the shortage of a standardized, end-to-end approaches for QoS assurance (between the end user, IoT devices and the cloud), the complexity of the integration of different layers, and the presence of a plethora of QoS constraints and parameters at each layer. We expect that the traditional way of QoS assurance will not be sufficient [20].

However, from the perspective of CloudIoT applications, network communication is an important but a smaller component. Parameters related to clouds (e.g. number of I/O operations and CPU throughput), devices (battery), network type, and application would have to be used in conjunction with network parameters (bandwidth, delay, and jitter) to determine the overall QoS of the CloudIoT applications. To realize the larger vision of CloudIoT, in particular the sensing-as-a-service paradigm, it is important to understand and carefully develop QoS metrics and corresponding SLA's that take into consideration the complexity introduced by each layer. We define CloudIoT QoS metric as (Relation 1):

\section{QoS=f (cloud QoS, network QoS, location, battery,..., N)}

Where $\mathrm{N}$ represents the total number of parameters. As can be observed from the equation, from end-to-end QoS is a multidimensional and a complex problem that needs to be carefully addressed [20]. 


\section{Conclusions}

Integration of cloud computing and the Internet of Things represents the next great leap forward in the future Internet. Application of this integration, which is called CloudIoT, valuable new paths will open for business and research. This integration can draw near future smart cities. However, the intersection between the Internet of Things, cloud and big data analysis systems still remains almost intact. Moreover, Cloud platforms need to be enhanced to support the rapid creation of applications, by providing domain specific programming tools and environments and seamless execution of applications, harnessing capabilities of multiple dynamic and heterogeneous resources, to meet QoS requirements of diverse users. The open issues of CloudIoT paradigm pertain mainly power and energy efficiency, SLA enforcement, pricing and billing, security and privacy.

\section{References}

1. In Lee, Kyoochun Lee, (2015) "The Internet of Things (IoT): Applications, investments, and challenges for enterprises", Business Horizons 58, 431-440, Elsevier.

2. A. Bott, W.Donato, V. Persico, A. Pescapé, (2016) "Integration of Cloud computing and Internet of Things: A survey", Future Generation Computer Systems 56, 684-700, Elsevier.

3. P. Mell, T. Grance, (2010) "Effectively and Securely Using the Cloud Computing Paradigm”, NIST, Information Technology Laboratory.

4. D. Leaf, (2010) "Overview: NIST Cloud Computing Efforts", NIST Senior Executive for Cloud Computing, NIST, Information Technology Laboratory.

5. Alfonso Castro, Víctor A. Villagrá, Beatriz Fuentes, and Begoña Costales, (2014) “A Flexible Architecture for Service Management in the Cloud", IEEE TRANSACTIONS ON NETWORK AND SERVICE MANAGEMENT, VOL. 11, NO. 1, MARCH.

6. http://blogs.idc.com/ie/?p=190

7. Stanoevska-Slabeva.K, Wozniak.T , Ristol.S, (2009) "Grid and Cloud Computing:A Business Perspective on Technology and Applications", Springer Science \& Business Media.

8. J. Zhou, T. Leppänen, E. Harjula, M. Ylianttila, T. Ojala, Ch. Yu, H. Jin, L. Tianruo Yang, "CloudThings: a Common Architecture for Integrating the Internet of Things with Cloud Computing", (2011), IEEE, 17th International Conference on Computer Supported Cooperative Work in Design.

9. A.K. Evangelos, D.T. Nikolaos, C.B. Anthony, (2011). "Integrating RFIDs and smart objects into a Unified Internet of Things architecture". Advances in Internet of Things.

10. L. Atzori, A. Iera, G. Morabito, (2010) “The Internet of Things: A survey”, Comput. Netw. 54 (15) 2787-2805. 
11. S.C.B. Intelligence, (2008) Disruptive civil technologies, in: Six Technologies with Potential Impacts on US Interests Out to 2025.

12. C. Dobre, F. Xhafa, (2014) "Intelligent services for big data science”, Future Generation Computer Systems. 37 267-281.

13. L. Yan, Y. Zhang, L.T. Yang, H. Ning, (2008) "The Internet of Things: From RFID to the Next-Generation Pervasive Networked Systems", CRC Press.

14. A. Alamri, W.S. Ansari, M.M. Hassan, M.S. Hossain, A. Alelaiwi, M.A. Hossain, (2013) "A survey on sensor-Cloud: architecture, applications, and approaches", Int. J. Distrib. Sens. Netw.

15. J. Gubbi, R. Buyya, S. Marusic, M. Palaniswami (2013) 'Internet of Things (IoT): A vision, architectural elements, and future directions', Future Generation Computer Systems, Vol. 29 No. 7, pp.1645 - 1660.

16. N. Alhakbani, M.M. Hassan, M.A. Hossain, M. Alnuem, (2014), "A framework of adaptive interaction support in Cloud-based Internet of Things (IoT) environment", in: Internet and Distributed Computing Systems, Springer, pp. 136-146.

17. S.K. Dash, S. Mohapatra, P.K. Pattnaik, (2010), "A survey on application of wireless sensor network using Cloud computing”, Int. J. Comput. Sci. Eng. Technol. 1 (4) 50-55.

18. B.P. Rao, P. Saluia, N. Sharma, A. Mittal, S.V. Sharma, (2012), "Cloud computing for Internet of Things \& sensing based applications”, in: Sensing Technology (ICST), Sixth International Conference on, IEEE, 2012, pp. 374-380.

19. A. Zaslavsky, C. Perera, D. Georgakopoulos, (2013), "Sensing as a service and big data". ArXiv Preprint arXiv:1301.0159.

20. P. Prakash Jayaraman, K. Mitra, S. Saguna, T. Shah, D. Georgakopoulos, R. Ranjan, "Orchestrating Quality of Service in the Cloud of Things Ecosystem", Volume 128 - No.7, 23. Quality of Service Architecture for Internet of Things and. Cloud Computing, 2015. 